\title{
Dynamics of Cancer-Immune System with External Treatment and Optimal Control
}

Fathalla A Rihan ${ }^{1 *}$ and Nouran F Rihan ${ }^{2}$

${ }^{1}$ Department of Mathematical Sciences, College of Science, United Arab Emirates University, UAE

${ }^{2}$ Faculty of Pharmacy, Clinical Program, Cairo University, Kasr El-Aini St., Cairo 11562, Egypt

\begin{abstract}
Herein, we present a mathematical model of tumour-immune interactions in presence of chemotherapy treatment. The model is governed by a system of delay differential equations with optimal control variables. A discrete time-delay is considered to justify the time-needed for the effector cells to develop a suitable response to the tumour cells. The control variables are included to justify the best treatment strategy with minimum side effects by reducing the production of new tumour cells and keeping the number of normal cells above the average of its carrying capacity. The numerical simulations show that the optimal treatment strategy reduces the load of tumour cells increases the effector cells after few days of therapy.
\end{abstract}

Keywords: Chemotherapy; Immune system; Mathematical modeling; Optimal control; Time-delay; Tumour

\section{Introduction}

Indeed, cancer remains a major cause of death in both developing and developed countries; see the statistics of Health World Organization (WHO) [1]. The term cancer represents numerous diseases that can affect any part of the body. It can similarly be referred to as malignant tumours and neoplasms. The unique procedure that cancer cells adopt includes their ability to multiply rapidly, exceeding their normal boundaries, and accordingly invading the adjacent organs and spreading the disease. Cancer is the most challenging disease to treat, although great research effort dedicated to revealing the relation between the tumour cells and immune system. The desired outcome from cancer treatment should be destruction of all cancer cells in the body, while maintaining the minimum level of healthy cells. Chemotherapy is one of the most highly adopted cancer treatment modalities, however, it was proved to be not the most convenient solution for tumour regression [2,3]. Progress is currently being made in attempting to eliminate tumour cells in the host by using an experimental form of immunotherapy $[4,5]$.

Immune system (IS) is responsible for monitoring substances that be normally present the body. Any new substance in the body that the IS does not recognize raises an alarm, causing the IS to attack it. Substances that cause the IS response are called antigen ${ }^{1}$. Cancer cells are different from normal cells in the body and usually have unusual substances on their outer surfaces that can act as antigens. However, cancer cells look very much like normal cells and are tricky, as they put almost a cape over themselves so that the IS cannot recognize the cancer cell [6].

The immunity (or defence against pathogens or cancer cells) has basically two categories: innate immunity and adaptive immunity [7]. The innate immune system represents a nonspecific (no memory) response to substance to which the body regards as foreign or potentially harmful. The innate system represents the first line of defence and quickly response to certain general signs of infection so that is unable to memorise the same pathogen should the body be exposed to it in the future. In most cases, the innate immunity can suffice to clear pathogens, but sometimes it is insufficient. In fact,

${ }^{1}$ Antigens may be contained within or on bacteria, viruses, other microorganisms, or cancer cells. pathogens possess ways to overcome the innate barrier and successfully canalize the host. The adaptive immunity is, however, very specific, it is called into action against pathogens that can evade or overcome innate immune defences. This takes time to develop, but the adaptive immune system 'remembers' antigens that it has previously encountered and responds immediately the next time they try to infect. There are two types of adaptive immune responses: humoral immunity, mediated by antibodies produced by B lymphocytes, and cell-mediated immunity, mediated by T-lymphocytes [8]. In fact, the innate immune response develops first and occurs on the order of minutes or hours; While adaptive immunity follows, innate and occurs on order of days or weeks. Each has inherit time-delay $\tau$ in their developments [9].

The interactions between tumour cells and immune system are very complex and need sophisticated models to describe such interactions [10]. Mathematical models, based on ordinary differential equations, delay differential equations, partial differential equations, have proven to be useful tools in analysing and understanding the IS interactions with viral, bacterial infections and cancerous cells. Several mathematical models have been suggested to describe the interactions of tumour and immune system over time (see e.g. the research papers [11-15]). Most of these papers describe the interactions between tumour cells and immune cells, tumour cells and normal cells alone [16], or consider the interactions of tumour-immune system with chemotherapy treatment $[2,3]$. In this paper, we provide a mathematical model of tumour-immune interactions in presence of chemotherapy treatment and optimal control variables. The control variables are incorporated to justify the best strategy of treatment and minimize side effects of the external treatment by reducing the production of new tumour cells, while keeping the number of normal cells above the average of its carrying capacity.

*Corresponding author: Rihan FA, Department of Mathematical Sciences, College of Science, United Arab Emirates University, Al Ain, 15551, UAE, Tel: + 971-3-76 73 333; E-mail: frihan@uaeu.ac.ae

Received September 09, 2016; Accepted October 27, 2016; Published October 29 2016

Citation: Rihan FA, Rihan NF (2016) Dynamics of Cancer-Immune System with External Treatment and Optimal Control. J Cancer Sci Ther 8: 257-261. doi: 10.4172/1948-5956.1000423

Copyright: ( 2016 Rihan FA, et al. This is an open-access article distributed under the terms of the Creative Commons Attribution License, which permits unrestricted use, distribution, and reproduction in any medium, provided the original author and source are credited. 


\section{Mathematical Models}

We first present a simple model that describes the dynamics of tumour cells, $\mathrm{T}(\mathrm{t})$, and activated effector cells, $\mathrm{E}(\mathrm{t})$, such as cytotoxic T-cells, macrophages, and natural killer cells that are cytotoxic to the tumour cells. We adopt a predator prey formulation of the tumour immunity problem as a battle between immune cells and tumour cells (predators and prey, respectively). The model takes the form

$$
\begin{aligned}
\frac{d E(t)}{d t} & =\sigma+\mathcal{F}(E(t), T(t))-\mu E(t) T(t)-\delta E(t), \\
\frac{d T(t)}{d t} & =\alpha T(t)(1-\beta T(t))-n E(t) T(t),
\end{aligned}
$$

with initial conditions: $\mathrm{E}(0)=\mathrm{E}_{0}, \mathrm{~T}(0)=\mathrm{T}_{0}$. Of course, the interaction between the effector and tumour cells leads to a reduction in the size of both populations with different rates, which are expressed by $-\mu \mathrm{E}(\mathrm{t}) \mathrm{T}(\mathrm{t})$ and $-\mathrm{nE}(\mathrm{t}) \mathrm{T}(\mathrm{t})$, respectively. Because of this interaction, the immune effector cells decrease the population of tumour cells at rate n. While, tumour cells infect some of the effector cells and therefore, the population of uninfected effector cells decreases at the rate $\mu$. If one considers $\mathrm{T}(\mathrm{t})$ as prey and $\mathrm{E}(\mathrm{t})$ as predator, then $\mathrm{F}(\mathrm{E}, \mathrm{T})$ is assumed to be Michaelis-Menton form, so that

$$
\mathcal{F}(E, T)=\frac{\rho E(t) T(t)}{\eta+T(t)}
$$

In this term, $\rho$ is the maximum immune response rate and $\eta$ is the steepness of immune response. The presence of the tumour cells virtually initiates the proliferation of tumour-specific effector cells to reach a saturation level parallel with the increase in the tumour populations. Hence, the recruitment function should be zero in the absence of the tumour cells, whereas it should increase monotonically towards a horizontal asymptote [17]. Here $\sigma$ represents the normal rate (not increased by the presence of the tumour) of the flow of adult effector cells into the tumour side (region). The source of the immune cells is outside of the system, so it is reasonable to assume a constant influx rate $\sigma$. Furthermore, in the absence of any tumour, the cells will die at a rate $\delta$.

To make the mathematical model closer to the reality, one can incorporate a discrete time-lag $\tau$ in the model (1) to justify the time required to stimulate the effector cells and develop a suitable response to the tumour cells, after recognizing the tumour cells. The new model with discrete time-lag takes the form

$$
\begin{aligned}
& \frac{d E(t)}{d t}=\sigma+\frac{\rho E(t-\tau) \mathrm{T}(t-\tau)}{\eta+\mathrm{T}(t-\tau)}-\mu E(t-\tau) T(t-\tau)-\delta E(t) \\
& \frac{d T(t)}{d t}=r_{2} T(t)(1-\beta T(t))-n E(t) T(t) .
\end{aligned}, t \geq 0
$$

This model is called delay differential equations (DDEs), in which we must provide initial functions:

$\mathrm{E}(\mathrm{t})=\psi_{1}(\mathrm{t})$ and $\mathrm{E}(\mathrm{t})=\psi_{2}(\mathrm{t})$ for all $\mathrm{t} \in[-\tau, 0]$, instead of initial values. In model (2), the presence of tumour cells stimulates the immune response, represented by the positive nonlinear growth term for the immune cells $\rho \mathrm{E}(\mathrm{t}-\tau) \mathrm{T}(\mathrm{t}-\tau) /(\eta+\mathrm{T}(\mathrm{t}-\tau))$. $\rho$ and $\eta$ are positive constants, $\tau \geq 0$ is the time delay that presents the time needed by the immune system to develop a suitable response after recognizing the tumour cells. The saturation term (Michaelis-Menton form) with the $\mathrm{E}(\mathrm{t})$ compartment and logistic term with the $\mathrm{T}(\mathrm{t})$ compartment are consoled. The presence of the tumour cells virtually initiates the proliferation of tumour-specific effector cells to reach a saturation level parallel with the increase in the tumour populations. Of course, the solution of DDEs model (2) should be bounded and nonnegative [18].

\section{Boundedness and Non-Negativity of the Model Solutions}

To show that the solutions of model (2) are bounded and remain non-negative in the domain of its application for sufficiently large values of time $t$, we recall the following Lemma:

\section{Lemma 1}

(Gronwall's Lemma [19]) Let $\mathrm{x}, \psi$ and $\chi$ be real continuous functions defined in $[a, b], \chi \geq 0$ for $t \in[a, b]$. We suppose that on $[a, b]$ we have the inequality

$$
x(t) \leq \psi(t)+\int_{a}^{t} \chi(s) x(s) d s .
$$

Then

$$
x(t) \leq \psi(t)+\int_{a}^{T} \chi(s) \psi(s) e^{\left(\int_{a}^{t} \chi(\xi) d \xi\right)} d s \text { in }[\mathrm{a}, \mathrm{b}] .
$$

Therefore, we arrive at the following Proposition:

\section{Proposition 1}

Let $(\mathrm{E}(\mathrm{t}), \mathrm{T}(\mathrm{t}))$ be a solution of system $(2)$, then $\mathrm{E}(\mathrm{t})<\mathrm{M}_{1}$ and $\mathrm{T}(\mathrm{t})$ $<\mathrm{M}_{2}$ for all sufficiently large time $\mathrm{t}$, where

$$
\begin{aligned}
& M_{1}=E(0)+\frac{\sigma}{\delta} \exp (\delta t)+\int_{0}^{t}\left[\rho e^{\delta(\tau+s)}\left(E(0)+\frac{\sigma}{\delta} e^{\delta s}\right) \exp \left(\int_{s}^{t} \rho e^{\delta(\tau+\xi)} d \xi\right)\right] d s, \\
& M_{2}=\max \left(\frac{1}{\beta}, T(0)\right) .
\end{aligned}
$$

For the proof, we refer to [9].

\section{Corollary 1}

If $\frac{\rho}{\eta+T} \geq \mu$, then the solutions (E,T) for model (2) are non-negative for any non-negative initial condition. However, if $\frac{\rho}{\eta+T}<\mu$, then there exist non-negative initial conditions such that $\mathrm{E}(\mathrm{t})$ becomes negative in a finite time interval.

\section{Model with Chemotherapy and Control}

To include external chemotherapy in model (2), we should consider extra two variables namely amount of chemotherapy $u(t)$ and normal cells $\mathrm{N}(\mathrm{t})$ with two control variables $\mathrm{v}(\mathrm{t})$ and $\mathrm{w}(\mathrm{t})$ (Figure 1 ). We assume the homogeneity of the tumour cells, then the model takes the form

$$
\begin{aligned}
& \frac{d E(t)}{d t}=w(t) \sigma+\frac{\rho E(t-\tau) \mathrm{T}(t-\tau)}{\eta+\mathrm{T}(t-\tau)}-\mu E(t-\tau) T(t-\tau)-\delta E(t)-\mathrm{F}_{1}(\mathrm{u}) \mathrm{E}(\mathrm{t}), \\
& \frac{d T(t)}{d t}=r_{2} T(t)\left(1-\beta_{1} T(t)\right)-n E(t) T(t)-\mathrm{c}_{1} \mathrm{~N}(\mathrm{t}) \mathrm{T}(\mathrm{t})-\mathrm{F}_{2}(\mathrm{u}) \mathrm{T}(\mathrm{t}), \\
& \frac{d N(t)}{d t}=r_{3} N(t)\left(1-\beta_{2} N(t)\right)-c_{2} T(t) N(t)-F_{3}(u) N(t), \\
& \frac{d u(t)}{d t}=v(t)-d_{1} u(t) .
\end{aligned}
$$

The drug kills all types of cells, with different killing rate for each type of cells: $F_{i}(u)=a_{i}\left(1-e^{-u}\right)$ is the fraction cell kill for a given amount of drug, $u(t)$, at the tumour site. The parameters $a_{1}, a_{2}$, and $a_{3}$ are the three different response coefficients. $v(t)$ represents the amount of dose that is injected into the system, while $d_{1}$ is the decay rate of the drug once it is injected. In this case, the quantity we will control directly 


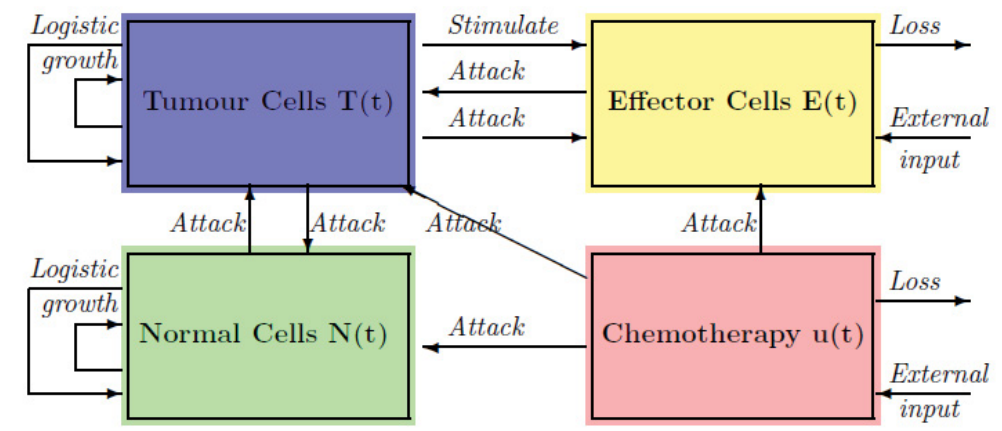

Figure 1: Schematic diagram of interaction of tumour cells, immune cells, and normal cells in the presence of chemotherapy treatment.

is not $\mathrm{u}(\mathrm{t})$, but $\mathrm{v}(\mathrm{t})$. The tumour cells and normal cells are modelled by a logistic growth law, with parameters $r_{i}$ representing the growth rate of two types of cells: $i=2$ identifies the parameter associated with tumour, and $\mathrm{i}=3$ identifies the one associated with the normal tissue. $\beta_{1}$ and $\beta_{2}$ are the reciprocal carrying capacities of tumour cells and host cells respectively. The two terms $-c_{1} N(t) T(t)$ and $-c_{2} N(t) T(t)$ represent the competition between the tumour and host cells.

Let $C=C\left([-\tau, 0], R^{4}\right)$ be the Banach space of continuous functions mapping the interval $[-\tau, 0]$ into $\mathrm{R}^{4}$ with the topology of uniform convergence. It is easy to show that there exists a unique solution $(\mathrm{E}(\mathrm{t})$, $\mathrm{T}(\mathrm{t}), \mathrm{N}(\mathrm{t}), \mathrm{u}(\mathrm{t}))$ of system (3) with initial data $\left(\mathrm{E}_{0}, \mathrm{~T}_{0}, \mathrm{~N}_{0}, \mathrm{u}_{0}\right) \in \mathrm{C}$. For biological reasons, we assume that the initial data of system (3) satisfy $\mathrm{E}_{0} \geq 0, \mathrm{~T}_{0} \geq 0, \mathrm{~N}_{0} \geq 0, \mathrm{u}_{0} \geq 0$. For $\tau=0$, the model is reduced to ODEs model developed by de Pillis and Radunskaya in [20].

The main objective in developing chemotherapy treatment, in system (3), is to reach either tumour-free steady state or coexisting steady state in which the tumour cells' size is small, while the normal cells' size is closed to its normalized carrying capacity. To keep the patient healthy while killing the tumour, our control problem consists of determining the variables $\mathrm{v}(\mathrm{t})$ and $\mathrm{w}(\mathrm{t})$ that will maximize the amount of effector cells and minimize the number of tumour cells. We use cost functional of the control with a constraint that to keep normal cells above the average of its capacity. Therefore, our objective is to maximize the functional ([9]).

$$
\max _{v, w} J(v, w)=\int_{0}^{t_{f}}\left(E(t)-T(t)-\left[\frac{B_{v}}{2}[v(t)]^{2}+\frac{B_{w}}{2}[w(t)]^{2}\right]\right) d t,
$$

Where $B_{u}, B_{w}$ are, respectively, the weight factors that describe the patient's acceptance level of chemotherapy and immunotherapy with a constraint

$$
k\left(E, T, N, u, E_{\tau}, T_{\tau}, v\right)=N-0.75 \geq 0,0 \leq t \leq t_{f} .
$$

We are seeking optimal control pair $\left(\mathrm{v}^{*}, \mathrm{w}^{*}\right)$ such that

$$
J\left(v^{*}, w^{*}\right)=\max \{J(v, w):(v, w) \in W\},
$$

where $\mathrm{W}$ is, the control set defined by

$$
\begin{aligned}
& W=\{(v, w):(v, w) \text { piecewise continuous, such that } \\
& \left.0 \leq v(t) \leq v_{\max }<\infty, ; 0 \leq w(t) \leq w_{\max }<\infty, \forall t \in\left[0, \mathrm{t}_{f}\right]\right\} .
\end{aligned}
$$

The existence of optimal controls $v^{*}(t)$ and $w^{*}(t)$ for this model is guaranteed by standard results in optimal control theory [21]. Necessary conditions that the controls must satisfy are derived via Pontryagins Maximum Principle [22]. The optimal control problem given by expressions (3)-(7) is equivalent to that of minimizing the Hamiltonian (See the Appendix):

$$
\begin{aligned}
& \mathcal{H}=E-T-\frac{B_{v}}{2}[v(t)]^{2}-\frac{B_{w}}{2}[w(\mathrm{t})]^{2}+\lambda_{1} \frac{d E}{d t}+\lambda_{2} \frac{d T}{d t}+\lambda_{3} \frac{d N}{d t}+\lambda_{4} \frac{d u}{d t}+\gamma k \\
& \text { and } \gamma \geq 0 \text { with } \gamma(\tau) k(t)=0 \text {, Where } \\
& \gamma= \begin{cases}1 & \text { if } \mathrm{N}(\mathrm{t}) \leq 0.75, \\
0 & \text { otherwise. }\end{cases}
\end{aligned}
$$

A standard application of Pontryagins Maximum Principle leads to the following result:

Theorem 1: There exists an optimal pair $v^{\star}(t)$ and $w^{\star}(t)$ and corresponding solutions $\mathrm{E}^{\star}, \mathrm{T}^{\star}, \mathrm{N}^{\star}$ and $\mathrm{u}^{\star}$ and that minimizes $\mathrm{J}(\mathrm{u}(\mathrm{t})$, $\mathrm{w}(\mathrm{t}))$ over $\Omega$. The explicit optimal controls are connected to the existence of continuous specific functions $\lambda_{i}$ for $i=1,2,3$, 4 satisfying the adjoin system

$$
\begin{aligned}
\lambda_{1}^{\prime}(t)= & -1+\lambda_{1}(t)\left[\delta+a_{1}\left(1-e^{-u^{*}}\right)\right]+\lambda_{2}(t) n T^{*}+\lambda_{1}(t+\tau) \chi_{\left[0, t_{f}-\tau\right]}\left[\mu T^{*}-\frac{\rho T^{*}}{\eta+T^{*}}\right], \\
\lambda_{2}^{\prime}(t)=1+ & \lambda_{2}\left[-r_{2}+2 r_{2} \beta_{1} T^{*}+n E^{*}+c_{1} N^{*}+a_{2}\left(1-e^{-u^{*}}\right)\right]+ \\
& \lambda_{3} c_{2} N^{*}+\chi_{\left[0, t_{f}-\tau\right]} \lambda_{1}(t+T)\left[\frac{\rho E^{*} T^{*}}{\left(\eta+T^{*}\right)^{2}}-\frac{\rho E^{*}}{\eta+T^{*}}+\mu E^{*}\right], \\
\lambda_{3}^{\prime}(t)= & \lambda_{2} c_{1} T^{*}-\lambda_{3}\left(r_{3}-2 r_{3} \beta_{2} N^{*}-c_{2} T^{*}-a_{3}\left(1-e^{-u^{*}}\right)\right)-\gamma, \\
\lambda_{4}^{\prime}(t)= & -\lambda_{1}(t) a_{1} e^{-u^{*}} E^{*}+\lambda_{2}(t) a_{2} e^{-u^{*}} T^{*}+\lambda_{3}(t) a_{3} e^{-u^{*}} N^{*}+\lambda_{4}(t) d_{1},
\end{aligned}
$$

with transversality conditions

$$
\begin{aligned}
& \lambda_{i}\left(t_{f}\right)=0, \mathrm{i}=\{1,2,3,4\} \text { and } \\
& \chi_{\left[0, t_{f}-\tau\right]}= \begin{cases}1 & \text { if } \mathrm{t} \in\left[0, \mathrm{t}_{f}-\tau\right], \\
0 & \text { otherwise. }\end{cases}
\end{aligned}
$$

Furthermore, the following properties hold

$$
v^{*}=\min \left(v_{\max }, \frac{\lambda_{4}}{B_{v}}\right), w^{*}=\min \left(w_{\max }, \frac{\lambda_{1} s_{1}}{B_{w}}\right) .
$$

(See the Delay Models with Optimal Control)

\section{Discussion and Conclusion}

In this paper, we provided a mathematical model with memory (time-delay) and optimal control variables to describe the dynamics of tumour-immune interactions in presence of chemotherapy treatments. The time-delay has been considered to describe the time needed by the immune system to launch a suitable response after recognizing the non-self-cells or foreign bodies. The numerical approximations of the optimal control problem are carried out using forward and backward Euler's methods. Starting with an initial guess for the value of the controls on the time interval $\left[0, t_{f}\right]$, we solve the state system 

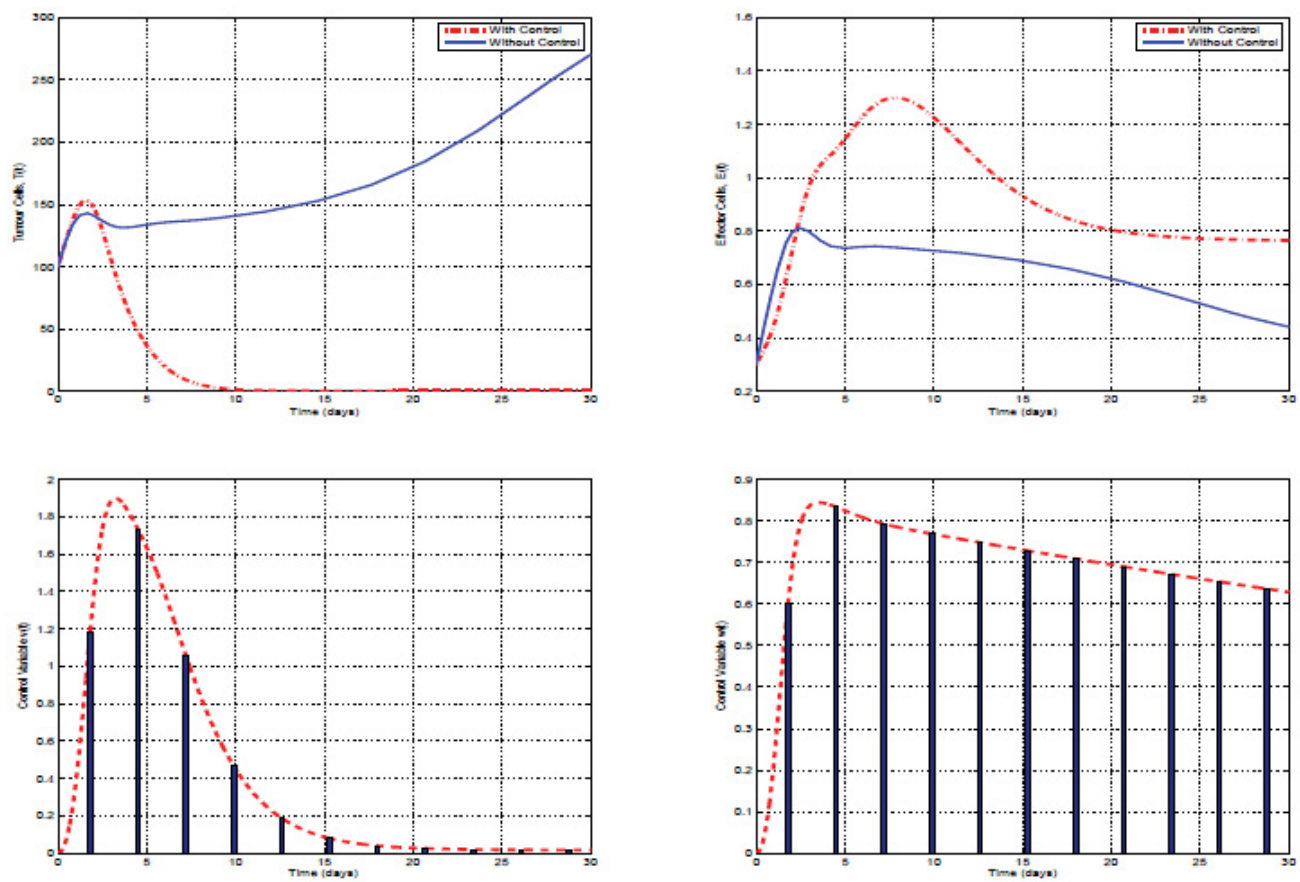

Figure 2: Numerical simulations of populations of the tumour cells and effector cells of system (3), before and after the chemotherapy treatment with controls $\mathrm{v}(\mathrm{t})$ and $\mathrm{w}(\mathrm{t})$. It shows that the tumour cells population can be eradicated in day 10 .

with control variables (3) using forward Euler's scheme. While, the adjoint system is solved using the solutions of the state system and the transversality conditions (9) backward in time. A Pontryagin-type maximum principle is derived, for optimal control problems with time-lag in the state variable. The control system is subject to a mixed control-state constraint to minimize the cost of treatment, reduce the tumour cells load, and keep the number of normal cells above $75 \%$ of its carrying capacity.

Figure 2 shows the impact of chemotherapy treatments (with optimal control) when we choose the parameter values in an unstable region $(\sigma=0.2, \rho=0.2$, and $\tau=1.5)$. The tumour cell population is growing up over time in the absence of chemotherapy, while the presence of treatment helps the immune system to keep the growth of the tumour cells under its control.

The Figure shows that the tumour cells can be eradicated at day 10 . The numerical simulations show the rationality of the model presented, which in some degree meets the natural facts.

The theoretical results presented in this paper convey a general insight to biologists and can help to gain better understanding of interaction mechanisms of tumour-immune system. They can be used to evaluate control strategies and applied for real cases in the future research work.

\section{Delay Models with Optimal Control}

Mathematical modelling with delay differential equations (DDEs) is widely used for analysis and predictions in epidemiology, immunology, physiology [23-27]. The time delays in these models consider a dependence of the present state of the modelled system on its history. In life, things are rarely so instantaneous; There is usually a propagation delay before the effects are felt. This situation can be modelled using a DDE.

$$
y^{\prime}(t)=f\left(y(t), \mathrm{y}\left(\mathrm{t}-\tau_{1}\right), y\left(t-\tau_{2}\right), \ldots, y\left(t-\tau_{d}\right), t\right), t \geq t_{0}
$$

where all the time-lags, $\tau_{\mathrm{i}}$, are assumed to be none negative functions of the current time t. Because of these delay terms it is no longer sufficient to supply an initial value, at time $t=t_{0}$, to completely define the problem. Instead, it is necessary to define the history of the state vector, $y(t)$, sufficiently far enough back in time from $t_{0}$ to ensure that all the delayed state terms,

$y\left(t-\tau_{i}\right)$, are always well defined. Thus, it is necessary to supply an initial state profile of the form:

$$
y(t)=\psi(t), t_{0}-\tau_{\max } \leq t<t_{0} \text {, and } y\left(t_{0}\right)=y_{0} .
$$

It should be noted that $\psi\left(\mathrm{t}_{0}\right)$ need not be the same as $\mathrm{y}_{0}$. This immediately introduces the possibility of a discontinuity in the state, $\mathrm{y}(\mathrm{t})$.

We mention here that there are many problems in biosciences (such as epidemics, harvesting, chemostat, treatment of diseases, physiological control, vaccination) which can be addressed within an optimal control framework for systems of DDEs [28-30]. However, the amount of real experience that exists with optimal control problems (OCPs) is still small. The DDE (12) can be converted into an optimal control problem by adding an $m$-dimensional control term $\mathrm{u}(\mathrm{t})$

$$
y^{\prime}(t)=f\left(y(t), y\left(t-\tau_{1}\right), y\left(t-\tau_{2}\right), \ldots, y\left(t-\tau_{d}\right), u(t), t\right)
$$

and a suitable objective functional (measure): $\mathrm{J}_{0}(\mathrm{u})$

$$
\begin{aligned}
& \text { Maximize } \\
& J_{0}(\mathrm{u})=\Phi_{0}(y(\mathrm{~T}))+\int_{0}^{t_{f}} \mathcal{L}\left(y(t), y\left(t-\tau_{1}\right), y\left(t-\tau_{2}\right), \ldots, y\left(t-\tau_{d}\right), u(t), t\right) d t,
\end{aligned}
$$

and subject to control constraint $\mathrm{a} \leq \mathrm{u}(\mathrm{t}) \leq \mathrm{b}$, and state constant $y(t) \leq c$, where $a$ and $b$ are the lower and upper bounds. The integrand, $\mathrm{L}(:)$ is called the Lagrangian of objective functional which is continuous in $\left[0, t_{f}\right]$. Additional equality or inequality constraint $(s)$ can be imposed in terms of $\mathrm{J}_{\mathrm{i}}(\mathrm{u})$. 
Citation: Rihan FA, Rihan NF (2016) Dynamics of Cancer-Immune System with External Treatment and Optimal Control. J Cancer Sci Ther 8: 257261. doi: $10.4172 / 1948-5956.1000423$

Pontryagin's Maximum Principle [22] gives necessary conditions that the control and the state need to satisfy, and introduces an adjoint function to affix to the differential equation to the objective functional. The necessary conditions needed to solve the optimal control problem are derived from the so called 'Hamiltonian' $\mathrm{H}$ which is given by the equation

$$
\mathcal{H}(t)=\mathcal{L}(:)+\lambda^{T}(t) f(:)
$$

Here, $\lambda^{\mathrm{T}}(\mathrm{t})$ is a vector of costate variables of the state variables $\mathrm{y}(\mathrm{t})$, which is the solution of the equation

$$
\lambda^{\prime}(t)=-\frac{\partial \mathcal{H}}{\partial y(t)}(t)-\chi_{\left[0, t_{f}-\tau\right]} \frac{\partial \mathcal{H}}{\partial y(t-\tau)}(t+\tau) \text { (Adjoin equation) }
$$

Where

$$
\chi_{\left[0, t_{f}-\tau\right]}= \begin{cases}1 & \text { if } \mathrm{t} \in\left[0, \mathrm{t}_{f}-\tau\right], \\ 0 & \text { otherwise. }\end{cases}
$$

Given the nonlinear Hamiltonian (16) in the controls $\mathrm{v}$ and $\mathrm{w}$, the process of solving the optimal control problem is to solve the state system (14) together with the adjoint equation (17) and the following conditions:

$$
\begin{aligned}
& \frac{\partial \mathcal{H}(t)}{\partial v}=\frac{\partial \mathcal{H}(t)}{\partial w}=0 \text { (Optimality conditions) } \\
& \lambda^{T}\left(t_{f}\right)=0 . \text { (Transversality conditions) }
\end{aligned}
$$

\section{Acknowledgement}

This work is generously supported by NRF Project (UAE). The authors would like to thank the reviewers for their constructive comments which improved the manuscript.

\section{References}

1. WHO (2016) WHO position paper on mammography screening, WHO Accessed on: $14^{\text {th }}$ Nov 2016.

2. De Pillis LG, Radunskaya A (2003) The dynamics of an optimally controlled tumor model: A case study. Math Comput Model 37: 1221-1244.

3. Swan GW (1985) Optimal control applications in the chemotherapy of multiple myeloma. IMA J Math Appl Med Biol 2: 139-160.

4. American Assocation for Cancer Research (2016) Special conference on tumor immunology and immunotherapy. Boston Marriott Copley Place Boston, Massachusetts, USA

5. Liu $Y$, Huang $H$, Saxena A, Xiang $J$ (2002) Intratumoral coinjection of two adenoviral vectors expressing functional interleukin-18 and inducible protein-10, respectively, synergizes to facilitate regression of established tumors. Cancer Gene Ther 9: 533-542.

6. National Cancer Institute. What is cancer?. Accessed on $14^{\text {th }}$ October 2016.

7. Janeway CA, Travers $P$, Walport M, Shlomchik MJ (2001) Immunobiology: The Immune System in Health and Disease. (5thedtn), Garland Science, New York.

8. Byrne HM, Alarcon T, Owen MR, Webb SD, Maini PK (2006) Modelling aspects of cancer dynamics: a review. Philos Trans A Math Phys Eng Sci 364: 1563-1578
9. Rihan FA, Abdelrahman D, Al-Maskari F, Ibrahim F (2014) Delay differential model for tumour-immune response with chemoimmunotherapy and optima control. Comput Math Methods Med 2014

10. Rihan FA, Hashish A, Al-Maskari F, Hussein MS, Ahmed E, et al. (2016) Dynamics of tumor-immune system with fractional-order. J Tumor Res 2:109.

11. Araujo RP, McElwain DL (2004) A history of the study of solid tumour growth the contribution of mathematical modelling. Bull Math Biol 66: 1039-1091.

12. Bellomo N, Li N, Maini $P$ (2008) On the foundations of cancer modeling: selected topics, speculations, and perspectives. Math Mod Methods Appl Sci 18: 593-646.

13. Chaplain MAJ (2008) Modelling aspects of cancer growth: Insight from mathematical and numerical analysis and computational simulation. In: Capasso V, Lachowicz M (Eds.) Multiscale Problems in the Life Sciences: From Microscopic to Macroscopic. Springer, Berlin.

14. Nagy JD (2005) The ecology and evolutionary biology of cancer: a review of mathematical models of necrosis and tumor cell diversity. Math Biosci Eng 2: $381-418$.

15. Roose T, Chapman S, Maini P (2007) Mathematical models of avascular tumor growth. SIAM Rev 49: 179-208.

16. Rihan FA, Safan M, Abdeen M, Abdel-Rahman D (2012) Mathematical modeling of tumor cell growth and immune system interactions. Int $\mathrm{J}$ Modern Physc 9: 95-111.

17. Villasana M, Radunskaya A (2003) A delay differential equation model for tumor growth. J Math Biol 47: 270-294.

18. Bodnar M, Forys U, Poleszczuk J (2011) Analysis of biochemical reactions models with delays. J Math Anal Appl 376: 74-83.

19. Halanay A (1966) Differential Equations, Stability, Ocsillations, Time lags. Academic press, New York.

20. de Pillis LG, Radunskaya A (2001) A mathematical tumor model with immune resistance and drug therapy: an optimal control approach. Comput Math Methods Med 3: 78-100.

21. Fleming WH, Rishel RW (1994) Deterministic and stochasitic optimal control. Springer-Verlag, New York.

22. Pontryagin LS, Boltyanski RV, Gamkrelidge RV, Mischenko EF (1962) The mathematical theory of optimal processes. John Wiley and Sons, NY.

23. Rihan FA (2000) Numerical treatment of delay dfferential equation in bioscience. University of Manchester, Manchester United.

24. Bocharov G, Rihan FA (2000) Numerical modelling in biosciences using delay differential equations. J Comput Appl Math 125: 183-199.

25. Fowler AC, Mackey MC (2002) Relaxation oscillations in a class of delay differential equations. SIAM J Appl Math 63: 299-323.

26. Nelson PW, Perelson AS (2002) Mathematical analysis of delay differentia equation models of HIV-1 infection. Math Biosci 179: 73-94.

27. Smith $H$ (2011) An introduction to delay differential equations with applications to the life sciences. Springer, New York.

28. Banks HT (1975) Modelling and control in biosciences. Springer, Berlin.

29. Kolmanovskii VB, Shaikhet L (1996) Control of systems with aftereffect, American Mathematical Society.

30. Smith SE (2005) Optimal control of delay differential equations using evolutionary algorithms. Complexity International 12: 1-10. 